\title{
Impending Cardiac Tamponade and Hemorrhagic Pleural Effusion as Initial Presentations of Incomplete Kawasaki Disease: A Case Report
}

\author{
Ye Ji Kim, M.D. ${ }^{1}$, Kyung Min Kim, M.D. ${ }^{1}$, Jae Young Lee, M.D., Ph.D. ${ }^{1}$, Ji Hong Yoon, M.D., Ph.D. ${ }^{1}$, \\ Dae Chul Jeong, M.D., Ph.D. ${ }^{1}$, Woo Young Park, M.D. ${ }^{2}$, Gi Beom Kim, M.D., Ph.D. ${ }^{2}$ \\ ${ }^{1}$ Department of Pediatrics, Seoul St. Mary's Hospital, College of Medicine, The Catholic University of Korea, ${ }^{2}$ Department of Pediatrics, Seoul \\ National University Children's Hospital, Seoul, Korea
}

\begin{abstract}
Kawasaki disease (KD) is an acute febrile illness that is characterized by systemic inflammation usually involving medium-sized arteries and multiple organs during the acute febrile phase, leading to associated clinical findings. The diagnosis is based on the principal clinical findings including fever, extremity changes, rash, conjunctivitis, oral changes, and cervical lymphadenopathy. However, KD diagnosis is sometimes overlooked or delayed because other systemic organ manifestations may predominate in acute phase of KD. As a cardiovascular manifestation, an acute pericarditis usually shows a small pericardial effusion, but large pericardial effusion showing clinical signs of cardiac tamponade is very rare. Here, we described a case of incomplete KD presenting with impending cardiac tamponade, and recurrent fever and pleural effusion. (J Rheum Dis 2020;27:68-72)
\end{abstract}

Key Words. Mucocutaneous lymph node syndrome, Pleural effusion, Cardiac tamponade

\section{INTRODUCTION}

Kawasaki disease (KD) is an acute, self-limiting febrile illness that is characterized by systemic inflammation involving all medium-sized arteries and multiple organs during the acute febrile phase, leading to associated clinical findings [1]. The diagnosis of $\mathrm{KD}$ is based on the presence of principal clinical findings: fever, extremity changes, rash, conjunctivitis, oral changes, and cervical lymphadenopathy [1]. However, the diagnosis of $\mathrm{KD}$ is sometimes overlooked or delayed because other systemic organ manifestations may precede such principal clinical features of $\mathrm{KD}$, especially in early infant and older children [2]. Common clinical scenarios mistakenly diagnosed before the diagnosis of KD are urinary tract infection (pyuria), aseptic meningitis (irritability and a culture-negative pleocytosis of the cerebrospinal fluid), bac- terial adenitis (retropharyngeal phlegmon), and bacterial sepsis (septicemic shock) [3]. Cardiovascular manifestations may include myocarditis, pericarditis, valvular regurgitation, shock, and coronary artery abnormalities [3]. Regarding pericarditis, echocardiographic findings of a small pericardial effusion are common, but pericardial effusion large enough to manifest clinical signs of cardiac tamponade is very rare $[4,5]$. Here, we describe a case of incomplete KD presenting with impending cardiac tamponade and hemorrhagic pleural effusion.

\section{CASE REPORT}

A previously healthy 10-month-old girl visited the emergency room due to high fever for 6 days, diarrhea, and respiratory distress. The past medical history was unremarkable. On arrival, the patient looked acutely ill

\footnotetext{
Received : September 30, 2019, Revised : October 22, 2019, Accepted : October 31, 2019

Corresponding to : Ji Hong Yoon (iD http://orcid.org/0000-0001-8498-2352

Department of Pediatrics, Seoul St. Mary's Hospital, College of Medicine, The Catholic University of Korea, 222 Banpo-daero, Seocho-gu, Seoul 06591, Korea. E-mail : sirbe@naver.com
}

Copyright (c) 2020 by The Korean College of Rheumatology. All rights reserved.

This is an Open Access article, which permits unrestricted non-commerical use, distribution, and reproduction in any medium, provided the original work is properly cited. 
and showed tachypnea with chest wall retraction. The blood pressure was $72 / 52 \mathrm{mmHg}$, heart rate $167 / \mathrm{min}$, respiratory rate 43 breaths/min, and the oxygen saturation $86 \% \sim 88 \%$. Initial laboratory findings showed white blood cell (WBC) count of $33.6 \times 10^{9} / \mathrm{L}$ (neutrophils $84.5 \%$, lymphocytes $13 \%$ ), erythrocyte sedimentation rate (ESR) of $43 \mathrm{~mm} / \mathrm{h}$ (normal range $<20$ ), and C-reactive protein of $31.47 \mathrm{mg} / \mathrm{dL}$ (normal range $<1.0$ ). The blood urea nitrogen and creatinine were 65 $\mathrm{mg} / \mathrm{dL}$ and $1.02 \mathrm{mg} / \mathrm{dL}$, respectively. Serum pro-brain natriuretic peptide level (BNP) was $13,835 \mathrm{pg} / \mathrm{mL}$. Urine analysis showed pyuria (20 29 WBCs per high-power field and negative nitrate test). Electrocardiogram showed ST elevation in leads $\mathrm{V}_{4-6}$. Chest radiography showed massive cardiomegaly with pleural effusion in the right thoracic cavity (Figure 1). Echocardiography revealed a large amount of pericardial effusion with impending cardiac tamponade, but normal ventricular systolic function and coronary arteries without giant aneur-

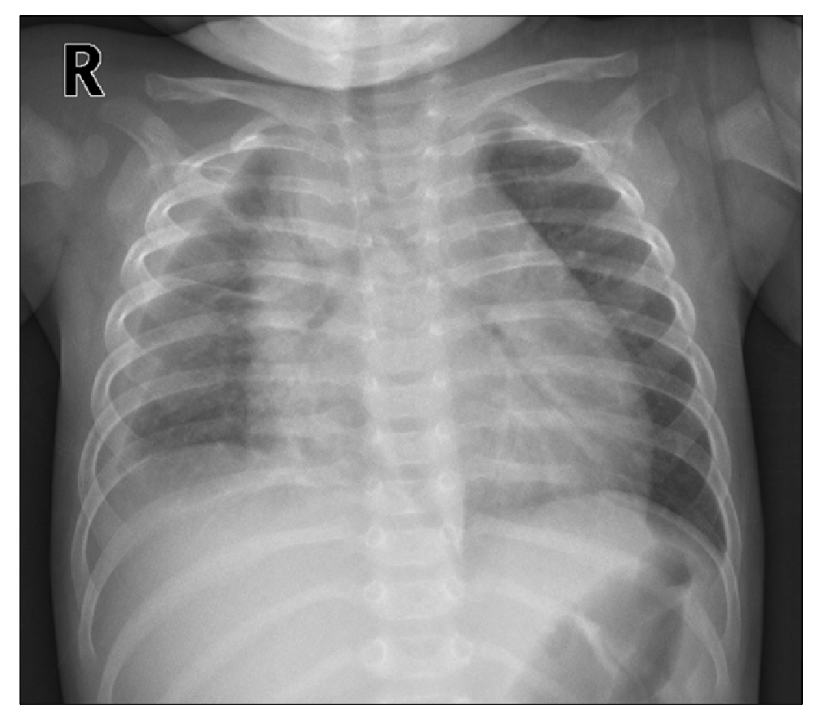

Figure 1. Frontal view of the chest radiograph shows cardiomegaly and pleural effusion at right thorax. ysm and aneurysmal rupture (Figure 2). Under the impression of acute pericarditis with impending cardiac tamponade and sepsis, an emergency pericardiocentesis was performed and serosanguinous fluid was drained. The results of pericardial fluid analysis were as follows: red blood cell count $300 \times 10^{6} / \mathrm{L}$, WBC count $570 \times 10^{9} / \mathrm{L}$ with $85 \%$ of neutrophils, lactate dehydrogenase (LDH) 26,900 IU/L (serum LDH 1,245 IU/L), and protein 3.87 $\mathrm{g} / \mathrm{dL}$ (serum protein $5.1 \mathrm{~g} / \mathrm{dL}$ ). Exudative pericardial effusion was diagnosed based on pericardial fluid to serum protein ratio $>0.5$ and pericardial fluid to serum LDH $>$ 0.6 (Light's criteria). In addition, the fluid drained through thoracentesis was hemorrhagic (red blood cell count $9,920 \times 10^{6} / \mathrm{L}$, the ratio of pleural fluid to serum LDH >0.6). Empirical antibiotics (cefotaxime and vancomycin) were administrated intravenously. Culture studies from blood, urine, and pericardial and pleural fluid failed to isolate any pathogen. Despite a regimen of multiple empirical antibiotics, high fever persisted. On the 19th day of fever, conjunctival injection and desquamation of both fingertips suggesting an incomplete $\mathrm{KD}$ developed. Follow-up laboratory tests were performed and met the criteria of supplemental laboratory findings supporting a diagnosis of incomplete KD (elevated ESR [25 mm/h] and CRP [20.73 mg/dL], low serum albumin [2.7 g/dL, reference range: $3.5 \sim 5.2]$, anemia for age [9.2 $\mathrm{g} / \mathrm{dL}]$, WBC count $\left[38.84 \times 10^{9} / \mathrm{L}\right]$, platelet count $\left[576 \times 10^{9} / \mathrm{L}\right]$ and sterile pyuria) [3]. Peripheral blood cell morphology showed that leukocytosis, neutrophilia, and normocytic normochromic anemia. There was an insufficient feature of macrophage activation syndrome (MAS) or systemic onset-juvenile idiopathic arthritis (SoJIA) in clinical course and follow-up laboratory test: Triglyceride (165 mg/dL, reference range: $40 \sim 200)$, ferritin $(210 \mathrm{ng} / \mathrm{mL}$, reference range: $26.1 \sim 287.6)$, fibrinogen $(243 \mathrm{mg} / \mathrm{dL}$, reference range: $160 \sim 350)$ (Table 1). Follow-up echocardiogram also showed a mild dilatation
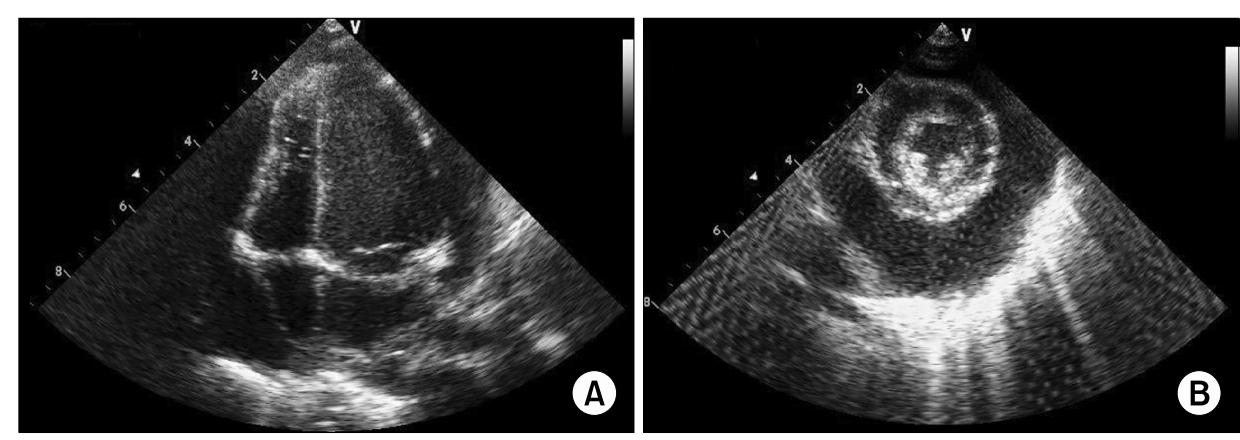

Figure 2. The echocardiographic apical four-chamber view (A) and parasternal short-axis view (B) show a massive pericardial effusion with impending cardiac tamponade. 
Ye Ji Kim et al.

Table 1. Laboratory values during treatment

\begin{tabular}{|c|c|c|c|c|c|c|c|c|c|c|c|c|c|c|}
\hline \multirow{2}{*}{ Day of fever } & \multicolumn{4}{|c|}{ 1st admission } & \multicolumn{4}{|c|}{ 2nd admission } & \multicolumn{3}{|c|}{ 3rd admission } & \multicolumn{3}{|c|}{ 4th admission } \\
\hline & 6 & 13 & $19 *$ & 22 & 25 & 28 & 31 & 34 & 39 & 43 & 48 & 53 & 60 & 67 \\
\hline WBC count $\left(10^{9} / \mathrm{L}\right)$ & 33.6 & 36.77 & 38.84 & 13.56 & 23.1 & 17.74 & 29.78 & 18.45 & 35.22 & 19.85 & 14.1 & 29.94 & 13.33 & 9.04 \\
\hline Lymphocytes (\%) & 13 & 26 & 25 & 40 & 22 & 24 & 39.6 & 41.4 & 15 & 31 & 56 & 17 & 50.1 & 62 \\
\hline Hemoglobin $(\mathrm{g} / \mathrm{dL})$ & 10 & 9 & 9.2 & 9.6 & 11.3 & 10.2 & 10.1 & 11.2 & 11.4 & 9.4 & 11 & 9.8 & 11.7 & 11.7 \\
\hline Platelet count $\left(10^{9} / \mathrm{L}\right)$ & 208 & 643 & 576 & 415 & 503 & 798 & 948 & 1,056 & 563 & 510 & 592 & 519 & 526 & 330 \\
\hline $\mathrm{ESR}(\mathrm{mm} / \mathrm{h})$ & 43 & 5 & 25 & 31 & 76 & 120 & 104 & 93 & 97 & 83 & 41 & 117 & 36 & 11 \\
\hline $\mathrm{CRP}(\mathrm{mg} / \mathrm{dL})$ & 31.47 & 5.8 & 20.73 & 4.38 & 13.96 & 27.73 & 6.32 & 2.06 & 8.51 & 4.82 & 0.17 & 23.44 & 0.13 & 0.43 \\
\hline Ferritin $(\mathrm{ng} / \mathrm{mL})$ & & 236 & 210 & 142.7 & & 348.8 & & & & & & & & \\
\hline Fibrinogen (mg/dL) & $>500$ & 225 & 243 & 239 & & 465 & & & & & & & & \\
\hline Triglyceride (mg/dL) & & 94 & 165 & 107 & & 126 & & & & & & 80 & & \\
\hline Albumin $(\mathrm{g} / \mathrm{dL})$ & 2.6 & 2.7 & 2.7 & 2.9 & 3.9 & 3.3 & 3.3 & 4.1 & 4.1 & 4.1 & & 3.9 & 4.2 & 4.1 \\
\hline $\mathrm{LDH}(\mathrm{IU} / \mathrm{L})$ & 1,245 & 701 & 586 & 536 & 299 & 203 & & & & & & & & \\
\hline AST (IU/L) & 59 & 22 & 22 & 26 & 43 & 22 & 20 & 28 & 28 & 21 & & 18 & 23 & 24 \\
\hline $\mathrm{ALT}(\mathrm{IU} / \mathrm{L})$ & 38 & 13 & 17 & 26 & 33 & 22 & 17 & 18 & 19 & 16 & & 9 & 8 & 7 \\
\hline
\end{tabular}

WBC: white blood cell, ESR: erythrocyte sedimentation rate, CRP: C-reactive protein, LDH: lactate dehydrogenase, AST: aspartate aminotransferase, ALT: alanine aminotransferase. *The day of diagnosis with incomplete Kawasaki disease.

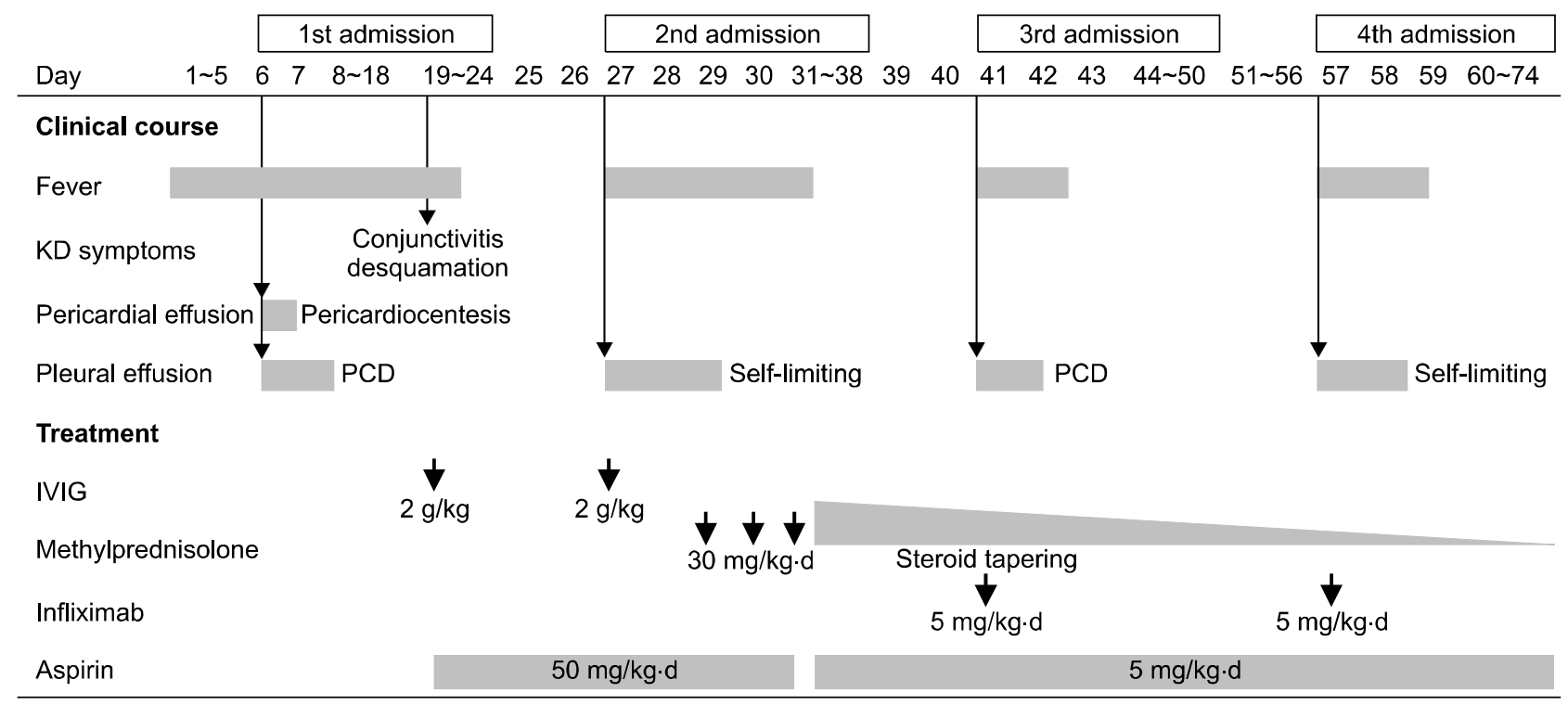

Figure 3. Clinical course and treatment of this patient. KD: Kawasaki disease, PCD: pigtail catheter drainage, IVIG: intravenous immunoglobulin.

of the right coronary artery $(2.4 \mathrm{~mm}$ in diameter; $\mathrm{Z}$ score $=2.7$ ) without pericardial effusion. Intravenous immunoglobulin (IVIG, $2 \mathrm{~g} / \mathrm{kg}$ ) and oral high-dose aspirin (50 mg/kg/day) were administered. Fever subsided on the 2nd day of IVIG treatment. The patient was discharged 4 days after IVIG treatment (Figure 3). Three days after the discharge, she was readmitted with a 24-hour history of high fever and pleural effusion. Despite the second dose of IVIG ( $2 \mathrm{~g} / \mathrm{kg})$, fever persisted (body temperature $>38.5^{\circ} \mathrm{C}$ ). Fever and pleural effusion were resolved after treatment with high dose methylprednisolone (Figure 3). After discharge, high fever and pleural effusion redeveloped twice, on the 41st and 57th days of illness, and laboratory findings also showed leukocytosis $\left(33.6 \times 10^{9} / \mathrm{L}\right.$ of WBC count) and elevated ESR $(43 \mathrm{~mm} / \mathrm{h})$ and CRP $(34.1 \mathrm{mg} / \mathrm{dL})$ on each occasion at the time of admission. After administration of infliximab $(5 \mathrm{mg} / \mathrm{kg}$ ), fever and pleural effusion subsided each time. After discharge following the 4th admission, the patient is doing well. Echocardiography performed 1 year after 
the illness showed normal coronary arteries and ventricular function.

\section{DISCUSSION}

$\mathrm{KD}$ is a systemic vasculitis that can affect various organs [1]. The diagnosis of classic $\mathrm{KD}$ is based on the presence of $\geq 5$ of 6 principal clinical criteria. Patients who have insufficient principal clinical features (fever $\geq 5$ days and 2 or 3 compatible clinical criteria) may be diagnosed with incomplete $\mathrm{KD}$, based on supportive laboratory findings or a positive echocardiogram [3]. However, KD diagnosis is sometimes delayed because other systemic manifestations may precede such principal clinical features of $\mathrm{KD}$. There are various unusual initial presentations that delay diagnosis of $\mathrm{KD}$, such as acute surgical abdomen [6], aseptic meningitis [7], cervical adenitis [8], and shock syndrome [9]. Zulian et al. [6] reported 10 cases of $\mathrm{KD}$ with acute surgical abdomen as an initial presentation, out of which 7 patients were diagnosed after surgical intervention. A prolonged fever and a culture-negative pleocytosis of cerebrospinal fluid suggestive of aseptic meningitis in a neonate may lead to an oversight of the diagnosis of $\mathrm{KD}$, because enteroviral meningitis is common in this age group [7]. Kanegaye et al. [8] reported that cervical adenitis and fever were initial presentations in 57 $\mathrm{KD}$ patients and suggested that incomplete $\mathrm{KD}$ should be considered in patients with cervical adenitis unresponsive to empirical antibiotics. In this case, incomplete $\mathrm{KD}$ was suspected and diagnosed only after development of conjunctivitis and desquamation on the fingertips on the 13th day of hospitalization (the 19th day of fever).

Because clinical criteria are used to diagnosis of $\mathrm{KD}$, patients who have insufficient principal clinical features tend to make early diagnosis difficult [3]. Moreover KD, SoJIA and MAS have similarity that clinical features and laboratory findings tend to overlap [10]. Therefore, the differential diagnosis of incomplete KD presenting especially unusual manifestation has diagnostic dilemma and clinical challenging, which often can be delay in diagnosis $[2,10]$. In our case, we tried to rule out infectious disease, drug-related polyserositis and connective tissue disease. According to the laboratory finding including bacterial cultures and virus studies, infectious disease could be excluded. The absence of signs of arthritis during 1 year follow-up, negative study of auto-immune antibodies (anti-nuclear antibody, rheumatoid factor), and other laboratory features (ferritin, triglyceride, fibrinogen, WBC count, platelet, etc.) were insufficient to diagnose of connective tissue disease especially SoJIA and MAS $[10,11]$.

$\mathrm{KD}$ with massive pericardial effusion showing clinical signs of cardiac tamponade is very rare. There have been several reports on hemorrhagic pleural and/or pericardial effusion in patients with $\mathrm{KD}[4,5,12,13]$. Voynow et al. [13] reported that 11 (1.83\%) out of $602 \mathrm{KD}$ patients presented with predominant pulmonary involvement. However, pericardial involvement is usually manifested as a small amount of pericardial effusion on the echocardiogram, and to our knowledge, only 2 cases with cardiac tamponade have been reported [4,5]. Our case showed an atypical course where massive pleural effusion and pericardial effusion with cardiac tamponade without giant aneurysm and aneurysmal rupture developed as initial presentations in an infant under 12 months.

Currently, first-line treatment of KD is a single dose of IVIG $(2 \mathrm{~g} / \mathrm{kg})$ and second-line treatment for IVIG-resistant KD patients may be a repeat dose of IVIG, high-dose methylprednisolone, or infliximab [3]. It is known that the levels of pro-inflammatory cytokines (interleukin [IL]-2, IL-6, IL-8, interferon- $\gamma$ and tumor necrosis factor $[\mathrm{TNF}]-\alpha$ ) and vascular endothelial growth factor (VEGF) are increased in KD $[14,15]$. Therefore, infliximab (anti-TNF- $\alpha$ monoclonal antibody) therapy is preferable to use in patients with KD refractory to IVIG [3]. Other monoclonal antibody therapy (anakinra, a recombinant, nonglycosylated form of the human IL-1 receptor antagonist) for treatment of refractory $\mathrm{KD}$ was reported [16]. Regarding the pathogenesis of pleural and pericardial effusion in $\mathrm{KD}$, it has been speculated that VEGF may play a role by increasing microvascular hyperpermeability [12]. Strunk et al. [17] reported that infliximab and glucocorticoids reduced VEGF in patients with rheumatoid arthritis. However, a study in KD showed that infliximab reduced pro-inflammatory cytokines but did not suppress VEGF in refractory KD patients [14]. Instead, Hamada et al. [12] reported a case that presented with marked pericardial effusion and pleural effusion with elevated VEGF, which were dramatically resolved after corticosteroid administration. In our case, recurrent fever and pleural effusion developed even during steroid administration, and showed acute response to infliximab therapy, suggesting its partial role in reducing the hyperpermeable state of $\mathrm{KD}$. Further study is needed to elucidate the possible roles and mechanisms of infliximab and corticosteroid in suppressing vascular hyperpermeability in $\mathrm{KD}$ patients. 


\section{SUMMARY}

$\mathrm{KD}$ is included on the list of important causes of MAS in childhood, and the large degree of overlap syndrome should be considered the possibility of similar relationships between refractory incomplete KD and MAS [18]. It is important to remember that in infants with unexplained persistent fever and irresponsive to empirical antibiotics, incomplete KD should be suspected. In addition, clinicians should be aware that cardiac tamponade and/or hemorrhagic pleural effusion may be initial clinical manifestations of incomplete KD.

\section{CONFLICT OF INTEREST}

No potential conflict of interest relevant to this article was reported.

\section{AUTHOR CONTRIBUTIONS}

Study concept and design: J.H.Y. Acquisition of data: K.M.K., D.C.J., W.Y.P., G.B.K. Analysis and interpretation of data: J.H.Y., Y.J.K. Writing manuscript: J.H.Y., Y.J.K., J.Y.L.

\section{REFERENCES}

1. Kawasaki T, Kousaki F. Fabrile oculo-oro-cutaneo-acrodesquamatous syndrome with or without acute non-suppurative cervical lymphadenitis in infancy and childfood: clinical observations of 50 cases. Jpn J Allergol 1967;16: 178-222, 225.

2. Minich LL, Sleeper LA, Atz AM, McCrindle BW, Lu M, Colan SD, et al.; Pediatric Heart Network Investigators. Delayed diagnosis of Kawasaki disease: what are the risk factors? Pediatrics 2007;120:e1434-40.

3. McCrindle BW, Rowley AH, Newburger JW, Burns JC, Bolger AF, Gewitz M, et al.; American Heart Association Rheumatic Fever, Endocarditis, and Kawasaki Disease Committee of the Council on Cardiovascular Disease in the Young; Council on Cardiovascular and Stroke Nursing; Council on Cardiovascular Surgery and Anesthesia; and Council on Epidemiology and Prevention. Diagnosis, treatment, and long-term management of Kawasaki disease: a scientific statement for health professionals from the American Heart Association. Circulation 2017;135:e92799.

4. Dahlem PG, von Rosenstiel IA, Lam J, Kuijpers TW. Pulse methylprednisolone therapy for impending cardiac tamponade in immunoglobulin-resistant Kawasaki disease. Intensive Care Med 1999;25:1137-9.
5. Ozdogu H, Boga C. Fatal cardiac tamponade in a patient with Kawasaki disease. Heart Lung 2005;34:257-9.

6. Zulian F, Falcini F, Zancan L, Martini G, Secchieri S, Luzzatto C, et al. Acute surgical abdomen as presenting manifestation of Kawasaki disease. J Pediatr 2003;142:731-5.

7. Rosenfeld EA, Corydon KE, Shulman ST. Kawasaki disease in infants less than one year of age. J Pediatr 1995;126: 524-9.

8. Kanegaye JT, Van Cott E, Tremoulet AH, Salgado A, Shimizu C, Kruk P, et al. Lymph-node-first presentation of Kawasaki disease compared with bacterial cervical adenitis and typical Kawasaki disease. J Pediatr 2013;162:1259-63, 1263.e1-2.

9. Thabet F, Bafaqih $\mathrm{H}$, Al-Mohaimeed S, Al-Hilali M, Al-Sewairi W, Chehab M. Shock: an unusual presentation of Kawasaki disease. Eur J Pediatr 2011;170:941-3.

10. Ravelli A, Minoia F, Davì S, Horne A, Bovis F, Pistorio A, et al.; Paediatric Rheumatology International Trials Organisation; Childhood Arthritis and Rheumatology Research Alliance; Pediatric Rheumatology Collaborative Study Group; Histiocyte Society. 2016 Classification criteria for macrophage activation syndrome complicating systemic juvenile idiopathic arthritis: a European League Against Rheumatism/American College of Rheumatology/Paediatric Rheumatology International Trials Organisation Collaborative Initiative. Ann Rheum Dis 2016;75:481-9.

11. Mizuta M, Shimizu M, Inoue N, Kasai K, Nakagishi $Y$, Takahara T, et al. Serum ferritin levels as a useful diagnostic marker for the distinction of systemic juvenile idiopathic arthritis and Kawasaki disease. Mod Rheumatol 2016;26: 929-32.

12. Hamada H, Terai M, Honda T, Kohno Y. Marked pleural and pericardial effusion with elevated Vascular Endothelial Growth Factor production: an uncommon complication of Kawasaki disease. Pediatr Int 2005;47:112-4.

13. Voynow JA, Schanberg L, Sporn T, Kredich D. Pulmonary complications associated with Kawasaki disease. J Pediatr 2002;140:786-7.

14. Hirono K, Kemmotsu Y, Wittkowski H, Foell D, Saito K, Ibuki K, et al. Infliximab reduces the cytokine-mediated inflammation but does not suppress cellular infiltration of the vessel wall in refractory Kawasaki disease. Pediatr Res 2009;65:696-701.

15. Jinkawa A, Shimizu M, Nishida K, Kaneko S, Usami M, Sakumura N, et al. Cytokine profile of macrophage activation syndrome associated with Kawasaki disease. Cytokine 2019;119:52-6.

16. Kone-Paut I, Cimaz R, Herberg J, Bates O, Carbasse A, Saulnier JP, et al. The use of interleukin 1 receptor antagonist (anakinra) in Kawasaki disease: a retrospective cases series. Autoimmun Rev 2018;17:768-74.

17. Strunk J, Bundke E, Lange U. Anti-TNF-alpha antibody Infliximab and glucocorticoids reduce serum vascular endothelial growth factor levels in patients with rheumatoid arthritis: a pilot study. Rheumatol Int 2006;26:252-6.

18. Latino GA, Manlhiot C, Yeung RS, Chahal N, McCrindle BW. Macrophage activation syndrome in the acute phase of Kawasaki disease. J Pediatr Hematol Oncol 2010;32:52731. 